\title{
Submitted: \\ 22.01.2020 \\ Accepted: 16.04.2020 \\ Published: \\ Ultrasound of the elbow with emphasis on the sonoanatomy of the distal biceps tendon and its importance for the surgical treatment of tendon lesions
} 15.06.2020

Keywords

ultrasound,

elbow,

biceps, tendon

\author{
Giorgio Tamborrini', Magdalena Müller-Gerbl², \\ Nicole Vogel ${ }^{3}$, David Haeni ${ }^{3}$
}

${ }^{1}$ UZR, Ultrasound Center and Institute for Rheumatology, EULAR Network of Imaging Center, Basel, Switzerland

${ }^{2}$ Institute of Anatomy, University of Basel, Basel, Switzerland

${ }^{3}$ LEONARDO, Hirslanden Clinic Birshof, Münchenstein, Baselland, Switzerland

Correspondence: Giorgio Tamborrini, UZR, Aeschenvorstadt 68, 4051 Basel, Switzerland; tel.: +412251010, e-mail:ultrasound@irheuma.com

DOI: $10.15557 / \mathrm{JoU} .2020 .0021$

\begin{abstract}
Degenerative or traumatic ruptures of the distal biceps tendon are less common than proximal lesions. Distal lesions lead to a significant loss of function with usually considerable discomfort for patients. Therefore, precise diagnostics using operator-dependent high-resolution musculoskeletal ultrasound with illustration of the extent of the affected tendon lesion are important for optimizing patient management. In this article, we discuss the precise highresolution musculoskeletal ultrasound and sonoanatomy of the distal biceps tendon and emphasize its importance for the surgical treatment of tendon lesions. In this review and pictorial essay, we first focus on the description of the precise anatomy and ultrasound anatomy of this clinically important region. Furthermore, we highlight different ultrasound scanning techniques for the correct assessment of the distal biceps tendon. Various approaches for optimal sonographic assessment of the distal biceps tendon have been suggested in the literature: the anterior approach, the lateral access, the medial access and the posterior approach. In the second part of the article, we focus on the evaluation of surgical repair techniques of distal biceps tendon lesions considering the extent of the rupture zone of the distal biceps tendon based on the ultrasound findings. Surgical techniques are explained from the orthopedic surgical point of view.
\end{abstract}

\section{Ultrasound of the distal biceps tendon}

The distal biceps tendon ( $\mathrm{dBT})$ is an elbow flexor, and it is involved in supination. The biceps muscle is innervated by the musculocutaneous nerve $(\mathrm{C} 5, \mathrm{C} 6)$. Performing ultrasound diagnostics of the $\mathrm{dBT}$, the patient ideally lies in the supine position on an examination couch with padding of the arm, if necessary. Alternatively, the examination can be performed in the sitting position. The arm is initially positioned in extension for static side comparison and dynamic analysis (flexion-extension, pronation-supination) of all structures on the anterior (cubital) elbow. The forearm is in the supination position. It is recommended to start the examination in the middle of the upper arm, and examine the structures continuously up to the ventral/anterior forearm in the transverse plane. This involves assessing both the osseous structures and the complete soft tissue. The easy-to-find trochlea humeri and the capitulum humeri with the hypoechoic hyaline cartilage serve as landmarks when assessing, for example, the soft tissue, the joint capsule or the joint cavity in the anterior elbow. The biceps tendon, the brachial artery, the median nerve, the deep brachial muscle, the ulnar-sided pronator teres muscle and the radial sided supinator muscle, the radial nerve (Fig. 1), 


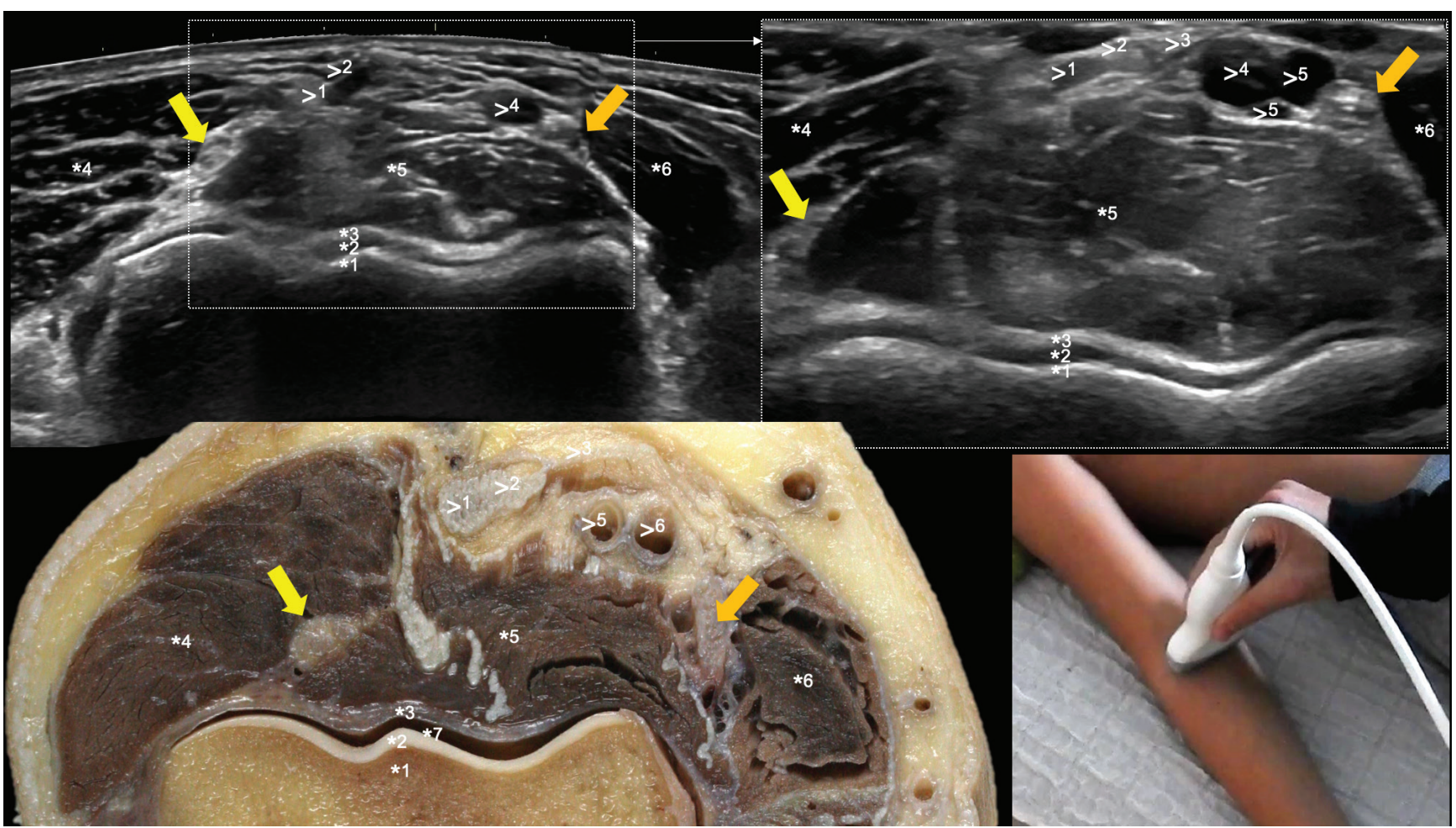

Fig. 1. Anterior elbow, transverse image in supination, B-mode, $11 \mathrm{MHz}$. *1 - cortical bone, trochlea humeri, *2 - hyaline cartilage, *3 joint capsule, *4 - brachioradialis muscle, $* 5$ - brachialis muscle, *6-pronator teres muscle, *7 - joint cavity, >1 - distal biceps tendon (long head), >2 - distal biceps tendon (short head), $>3$ - lacertus fibrosus, $>4-$ brachial artery, $>5-$ veins, yellow arrow - radial nerve, orange arrow - median nerve

and the anterior interosseous nerve can be assessed. The authors do not examine in consideration of probe positions, because we focus exclusively on the ultrasound image and orient ourselves to the anatomical landmarks in ultrasound images. The examination is dynamic and functional, so it must be documented on video (Video 1 - available at www. jultrason.pl).

The flat dBT is approximately 6 to $7 \mathrm{~cm}$ long, $6 \mathrm{~mm}$ wide, and $3 \mathrm{~mm}$ thick ${ }^{(1)}$. The distal biceps tendon moves obliquely from anterior to posterior, and from medial to lateral, with the $\mathrm{dBT}$ rotating $90^{\circ}$, so that the anterior surface points to the side. As the forearm moves from supination to pronation, the radial tuberosity shifts from medial to posterior position. The biceps tendon winds around the radius and compresses the bursa situated in between. The dBT is covered by an extrasynovial paratenon and by the bicipitoradial bursa. The bursa is normally not visible by high-resolution musculoskeletal ultrasound, unless it is filled with some amount of synovial fluid.

Various approaches for optimal sonographic assessment of the $\mathrm{dBT}$ have been suggested in the literature ${ }^{4)}$ :

- anterior approach (we do not recommend it for the completely distal sections, the reason being that it is often painful for the patient and not sensitive enough, with too many anisotropy artifacts)

- lateral access (through the brachio-radialis muscle and through the supinator muscle $=$ supinator window)
- medial access (through the pronator teres muscle = pronator window)

- posterior approach (through the anconeus muscle)

The dBT is most easily examined at the beginning of medial longitudinal in the supination position of the forearm, in an approx. 20 to $30^{\circ}$ flexion position in the elbow joint. We examine through the musculus pronator teres, with evaluation of the $\mathrm{dBT}$ up to the distal insertion at the radius. We recommend using the radius as a bony landmark. Generally, simple visualization of the distal biceps tendon at the radial insertion point is possible by visualizing the radial artery simultaneously with the radius (Fig. 2).

The assessment of the insertion at the radius below the supinator muscle is ideally performed by scanning through the supinator muscle. The forearm is maximally pronated, and the wrist is flexed. Both the lateral and posterior approaches are suitable for this purpose.

The insertion of the $\mathrm{dBT}$ is located at the radial tuberosity and a part at the fascia antebrachii (lacertus fibrosus). The entheseal medial insertion can be divided anatomically and sonographically into two sections. The distal tendon of the long head (lBT) inserts in the proximal section of the radial tuberosity, while the distal tendon of the short head (sBT) is located more distally (Fig. 3) ${ }^{(2)}$. In addition, the double tendon insertion allows an element of independent function of each section of the biceps ${ }^{(3)}$ : 


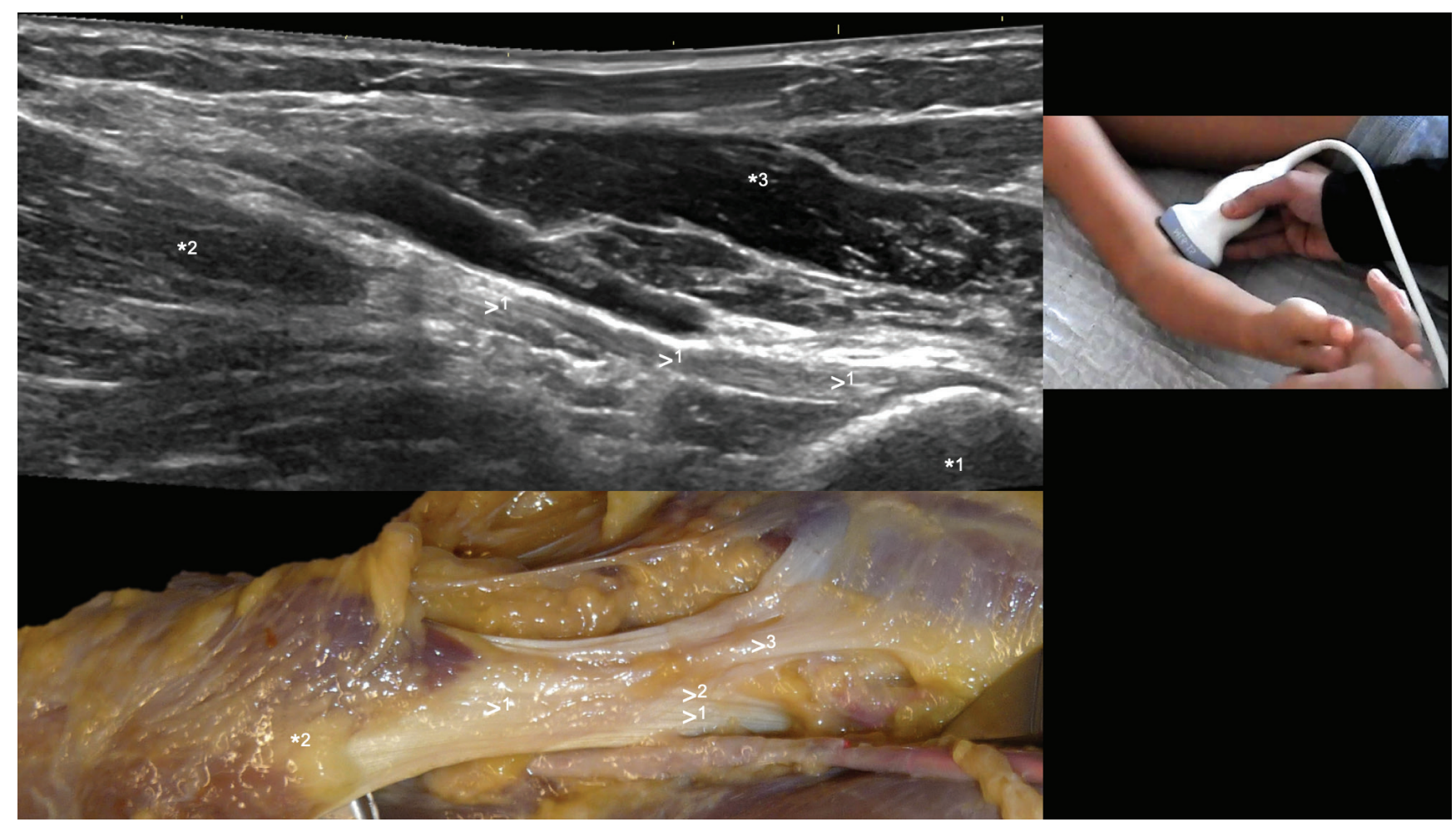

Fig. 2. Anterior/medial elbow, longitudinal image, B-Mode, $11 \mathrm{MHz}>1$-distal biceps tendon (long head), >2 - distal biceps tendon (short head), >3-lacertus fibrosus, *1-radius, *2 - biceps muscle, long head, *3-pronator teres muscle

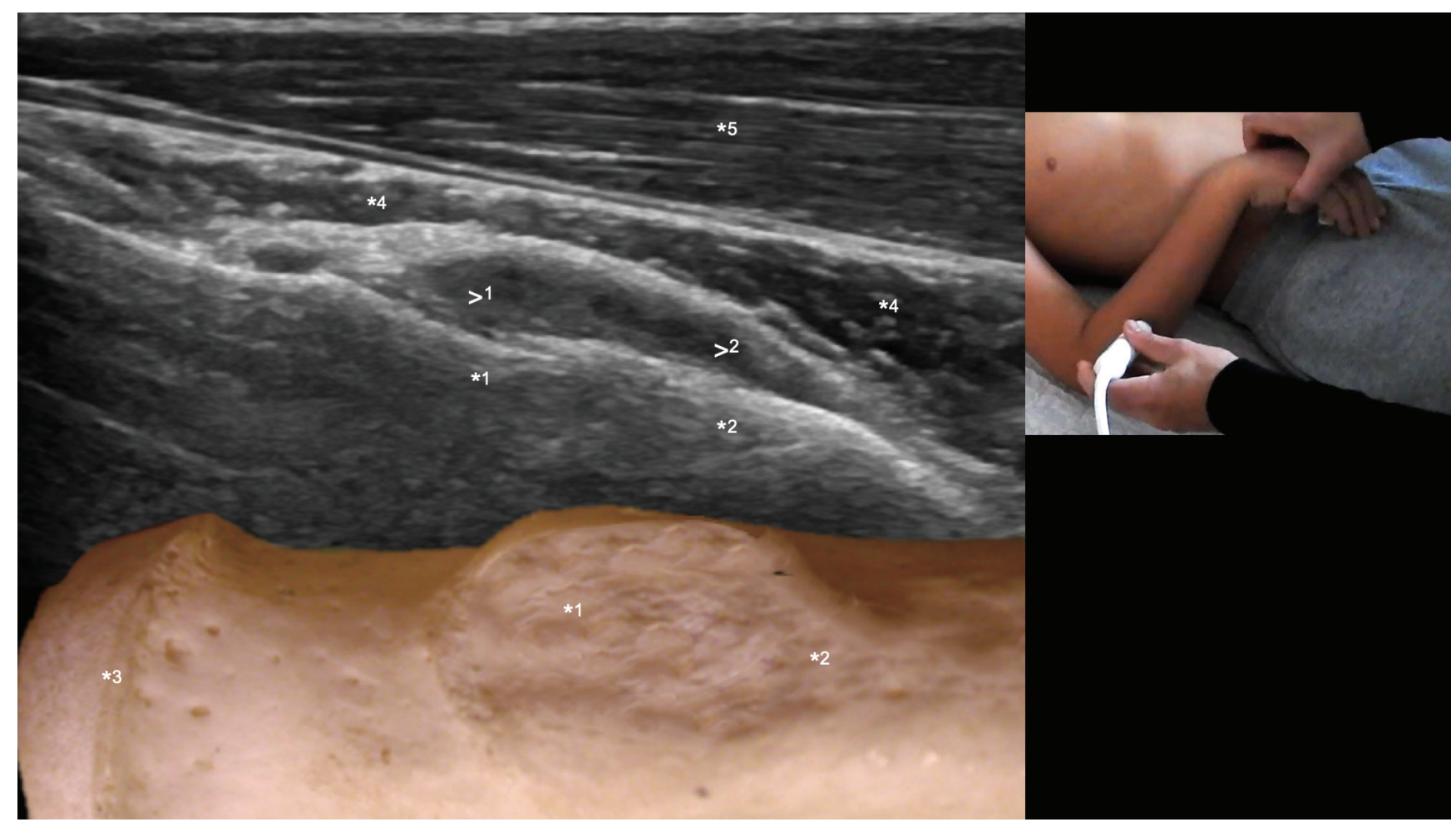

Fig. 3. Posterior/lateral elbow, longitudinal image in maximal pronation, B-Mode, $11 \mathrm{MHz}$. >1 - distal biceps tendon (long head), >2 distal biceps tendon (short head), *1 - insertion of the long head of the biceps tendon at the footprint/radial tuberosity, *2 - insertion of the short head of the biceps tendon at the footprint/radial tuberosity, *3-radial head, *4-supinator muscle, *5 - flexor carpi radialis muscle 


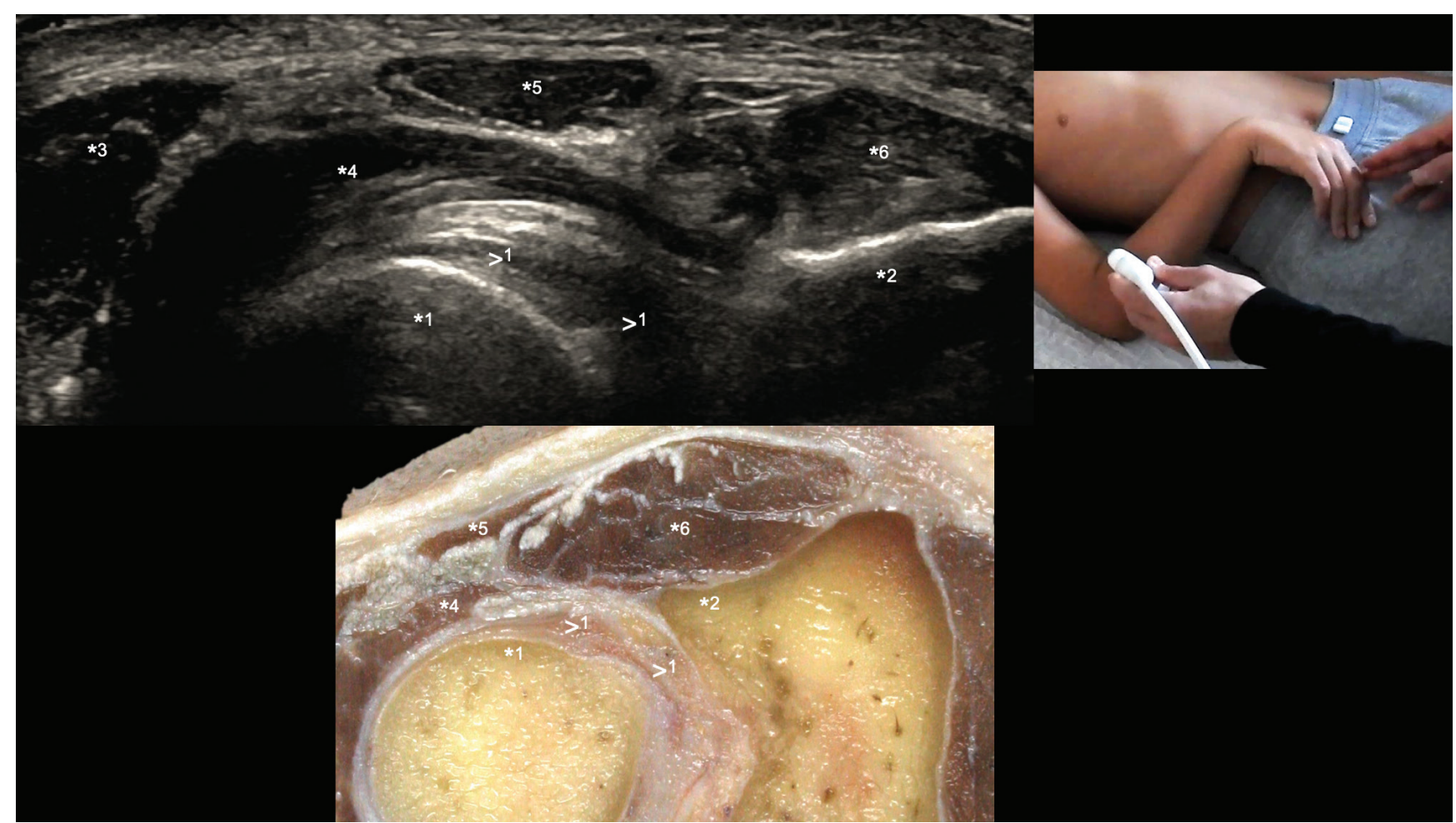

Fig. 4. Posterior/lateral elbow, transverse image in maximal pronation, B-Mode, $11 \mathrm{MHz}$. >1 - distal biceps tendon, *1 - insertion of the distal biceps tendon at the footprint / radial tuberosity, *2-ulna, *3-extensor digitorum communis muscle, *4-supinator muscle, *5-extensor carpi ulnaris muscle, *6-anconeus muscle

- the distally inserting sBT is a stronger flexor of the elbow

- the proximally inserting $1 \mathrm{BT}$ is a stronger supinator when viewed from the axis of rotation of the forearm

The lacertus fibrosus (part of the aponeurosis which usually develops at the height of the myotendinous junction, pulling towards the fascia antebrachii) originates from the dBT of the short head (Fig. 1) $)^{(3)}$. The lacertus fibrosus covers the median nerve and the brachial artery, and has the function of keeping the biceps tendon in the correct position.

The biceps tendon insertion is located on average approx. $23 \mathrm{~mm}$ distally to the articular edge of the radial head. The average length of the biceps tendon insertion at the tuberosity is approx. $21 \mathrm{~mm}$, with an average width of $7 \mathrm{~mm}$ (Fig. 2, Fig. 3, Fig. 4). The average total area of the enthesis (footprint) is about $108 \mathrm{~mm}^{2}$ (footprint of the long head: $48 \mathrm{~mm}^{2}$, footprint of the short head: $\left.60 \mathrm{~mm}^{2}\right)^{(1-3)}$.

Ruptures of the dBT are best visualized in the long axis, and they appear as an anechoic or hypoechogenic discontinuity of tendon fibers with or without retraction and surrounding hypoechoic fluid (e.g. inside the bicipitoradial bursa) or isoechoic hematoma (Fig. 5). The degree of tendon retraction is not necessarily an indicator of the condition of the lacertus fibrosus, so it must be assessed separately ${ }^{(4)}$. The detailed assessment of the biceps tendon footprint supports, due to high anatomical variation of the position and shape of the tuberosity and insertion of the long biceps tendon, among other things, surgical reconstruction (including bone tunnels, suture anchors) ${ }^{(5-7)}$.
We are convinced that the importance of sonography is underlined by the contributions of the elbow surgeons in the following section.

\section{Treatment options}

A partial or complete rupture of the dBT can be visualized by high-resolution musculoskeletal ultrasound or MR. In cases of partial tears (less than $50 \%$ affected) ${ }^{(5)}$, patients are usually treated conservatively ${ }^{(6,7)}$. However, there is a high failure rate (up to 56\%) in patients with partial ruptures involving more than $50 \%$ of the $\mathrm{dBT}$ and in patients with a high physical load and repetitive activities ${ }^{(8)}$. For this reason, surgical treatment of partial lesions involving more than $50 \%$ of the $\mathrm{dBT}$ is recommended.

In cases of complete rupture, open refixation is recommended in most patients. Surgically treated patients have a better function $^{(9-11)}$. Various factors should be considered when deciding on therapy, including the activity level, comorbidities, hand dominance, and risk-benefit ratio ${ }^{(10)}$. Conservative treatment is an option for patients with low functional demands and needs ("low functional demands") or with relevant comorbidities. These patients, however, show clinically relevant deficits in various activities ${ }^{(9)}$ e.g. $40 \%$ loss of supination power, and $30 \%$ loss of flexion power ${ }^{(12)}$.

A number of open and endoscopic surgical techniques have been described and suggested in the literature. The so-called open "single incision" technique is more widely 


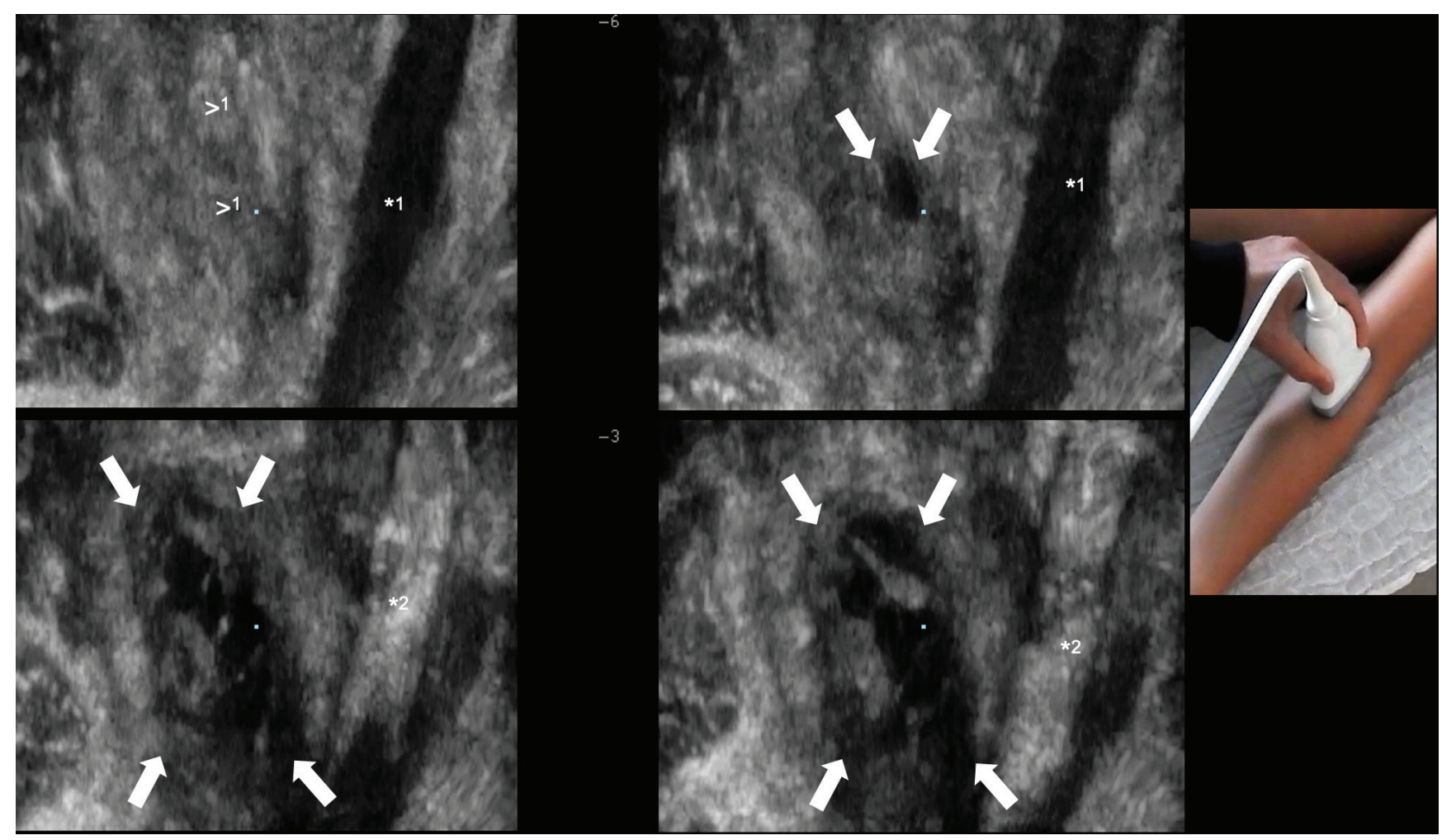

Fig. 5. Anterior elbow, coronar scan, B-mode, $11 \mathrm{MHz}$. *1-radial artery, *2-median nerve, $>1$ - normal fibers of the distal biceps tendon, white arrows - partial tear of the distal biceps tendon (arrows) with inhomogeneous bicipitoradial bursitis

used in Europe (Fig. 6). However, various biomechanical studies point to possible imperfect anatomical readaptation when using this technique. The "double incision" technique involves open refixation: the first anterior incision is made to locate the $\mathrm{dBT}$, and the second posterior incision is made to fix it to the radial tuberosity ${ }^{(13)}$. Endoscopic refixation techniques have also been reported ${ }^{(14)}$.

Intraoperative and postoperative complications may occur in up to $25 \%$ of cases ${ }^{(15)}$. A postoperative rupture is described in approximately $2.5 \%$ of cases ${ }^{(16)}$. Neuroapraxia

Tab. 1. Pathologies detectable by high-resolution ultrasound ${ }^{(18)}$

- Mechanical enthesopathy
- Inflammatory enthesitis
- Calcifications in the tendon or the enthesis
- Neovascularization, e.g. by means of B-flow examination
- Cubital bursitis (bizipitoradial bursitis)
- Interosseous bursitis
- Tendinosis (usually 1-2 cm proximal)
- Partial ruptures
- Complete ruptures
- Avulsions with osseous tearing
- Postoperative assessment with evaluation of tendon integrity, anchor
- position, suture position
- Metermination of tendon elasticity using elastography
- Secondary irritation caused by enthesophytes, osteophytes or by arti-
cular pathologies (synovitis, activated cubital arthrosis, synovial chon-
dromatosis, etc.)

of the N. cutaneus lateralis antebrachii (a sensitive branch of the N. musculocutaneus) can occur in up to $10 \%$ of cases with excessive traction during surgery. The complication is usually temporary, with restitutio ad integrum. Other complications, such as injury to the interosseus posterior nerve (PIN), heterotopic ossifications, and radioulnar synostoses, are possible. Heterotopic ossifications occur less commonly with the above mentioned "single incision" technique compared to the "double incision" technique (7\%), but are often clinically irrelevant ${ }^{(17)}$.

\section{Conclusions}

High-resolution musculoskeletal ultrasound with an axial and lateral resolution of up to $0.1 \mathrm{~mm}$ allows a differentiated assessment of the two parts of the distal biceps tendon, and thus the detection of different pathologies (Tab. 1) ${ }^{(18)}$. Due to high diagnostic accuracy of high-resolution musculoskeletal ultrasound, isolated lesions of one of the two components of the $\mathrm{dBT}$ can be distinguished, which may determine the clinical management of e.g. young patients from surgical to conservative treatment. A timely and accurate diagnosis allows earlier refixation, which in turn leads to better functional results with a low postoperative complication rate.

\section{Acknowledgment}

Considering "The Code of Ethics of the Word Medical Association" (Declaration of Helsinki), for experiments involving 


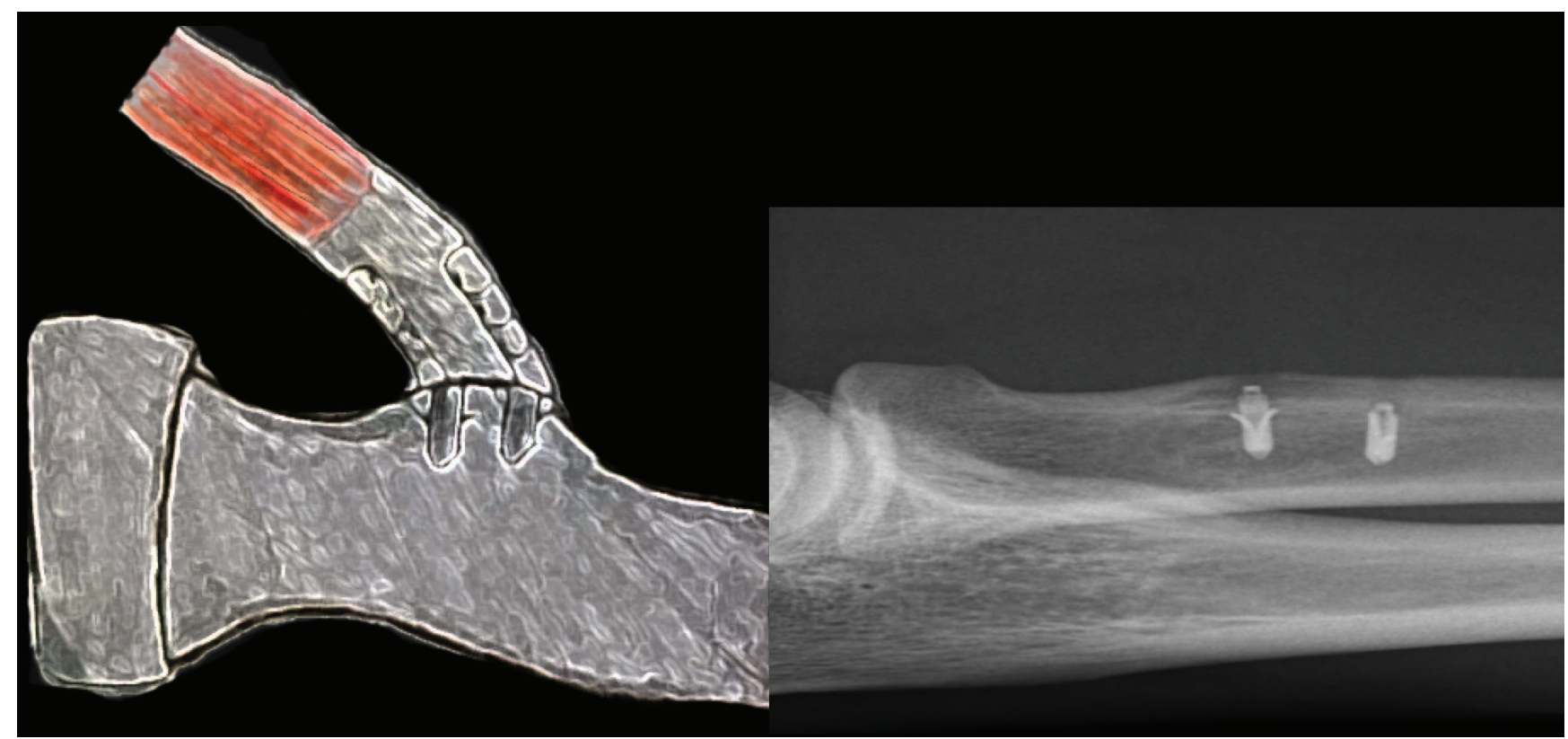

Fig. 6. Refixation of the distal biceps tendon: schematic illustration and postoperative $x$-ray showing the anchors at the radial tuberosity

humans, the samples were taken from body donors who contributed their body to science and research only to the departments.

All ultrasound images were taken by author 1, while anatomical images and sections were produced by authors 1 and 2.

\section{References}

1. Walton C, Li Z, Pennings A, Agur A, Elmaraghy A: A 3-dimensional anatomic study of the distal biceps tendon: implications for surgical repair and reconstruction. Orthop J Sports Med 2015;; 3: 2325967115585113.

2. Athwal GS, Steinmann SP, Rispoli DM: The distal biceps tendon: footprint and relevant clinical anatomy. J Hand Surg Am. 2007; 32: 1225-1229.

3. Eames MH, Bain GI, Fogg QA, van Riet RP: Distal biceps tendon anatomy: a cadaveric study. J Bone Joint Surg Am: 2007; 89: 1044-1049.

4. Konin GP, Nazarian LN, Walz DM: US of the elbow: indications, technique, normal anatomy, and pathologic conditions. Radiographics 2013; 33: E125-E147. Doi: 10.1148/rg.334125059.

5. de la Fuente J, Blasi M, Martínez S, Barceló P, Cachán C, Miguel M et al.: Ultrasound classification of traumatic distal biceps brachii tendon injuries. Skeletal Radiol 2018; 47: 519-532.

6. Stucken C, Ciccotti MG: Distal biceps and triceps injuries in athletes. Sports Med Arthrosc Rev 2014; 22: 153-163.

7. Dürr HR, Stäbler A, Pfahler M, Matzko M, Refior HJ: Partial rupture of the distal biceps tendon. Clin Orthop Relat Res 2000; (374): 195-200.

8. Bauer TM, Wong JC, Lazarus MD: Is nonoperative management of partial distal biceps tears really successful? J Shoulder Elbow Surg 2018; 27: 720-725.

9. Chillemi C, Marinelli M, De Cupis V: Rupture of the distal biceps brachii tendon: conservative treatment versus anatomic reinsertion-clinica and radiological evaluation after 2 years. Arch Orthop Trauma Surg 2007; 127: 705-708.

\section{Conflict of interest}

The authors do not report any financial or personal connections with other persons or organizations which might negatively affect the contents of this publication and/or claim authorship rights to this publication.

10. Savin DD, Watson J, Youderian AR, Lee S, Hammarstedt JE, Hutchinson MR et al:: Surgical management of acute distal biceps tendon ruptures. J Bone Joint Surg Am 2017; 99: 785-796.

11. Sutton KM, Dodds SD, Ahmad CS, Sethi PM: Surgical treatment of distal biceps rupture. J Am Acad Orthop Surg 2010; 18: 139-148.

12. Morrey BF, Askew LJ, An KN, Dobyns JH: Rupture of the distal tendon of the biceps brachii. A biomechanical study. J Bone Joint Surg Am 1985; 67: 418-421.

13. Wirth J, Bohnsack M: Distale Bizepssehnenruptur und Refixation der Sehne über zwei Zugänge. Oper Orthop Traumatol 2003; 15: 415.

14. Legg AJ, Stevens R, Oakes NO, Shahane SA: A comparison of nonoperative vs. Endobutton repair of distal biceps ruptures. J Shoulder Elbow Surg 2016; 25: 341-348.

15. Watson JN, Moretti VM, Schwindel L, Hutchinson MR: Repair techniques for acute distal biceps tendon ruptures: A systematic review. J Bone Joint Surg Am 2014; 96: 2086-2090.

16. Amin NH, Volpi A, Lynch TS, Patel RM, Cerynik DL, Schickendantz MS et al.: Complications of distal biceps tendon repair: a meta-analysis of single-incision versus double-incision surgical technique. Orthop J Sports Med 2016; 4: 2325967116668137.

17. Behun MA, Geeslin AG, O'Hagan EC, King JC: Partial tears of the distal biceps brachii tendon: a systematic review of surgical outcomes. J Hand Surg Am 2016; 41: e175-189.

18. Tamborrini G, Bruyn GAW, Staerkle-Baer A: Musculoskeletal Ultrasound Sonoanatomy Guidelines. Irheuma.com. ISBN 978-3-7460$6295-2$ 